\title{
Identification of Potocki-Lupski syndrome in patients with developmental delay and growth failure
}

\author{
Sujin Jun ${ }^{1 \oplus}$, Yena Lee ${ }^{1 \oplus}$, Arum Oh $^{1 \oplus}$, Gu-Hwan Kim ${ }^{2 \oplus}$, Eulju Seo $^{2 \oplus}$, Beom Hee Lee ${ }^{1,2}$, Jin-Ho Choi ${ }^{1,2} \oplus$, and Han-Wook Yoo ${ }^{1,2, * \oplus}$ \\ ${ }^{1}$ Department of Pediatrics, Asan Medical Center Children's Hospital, University of Ulsan College of Medicine, Seoul, Korea \\ ${ }^{2}$ Medical Genetics Center, Asan Medical Center Children's Hospital, University of Ulsan College of Medicine, Seoul, Korea
}

Purpose: Potocki-Lupski syndrome (PTLS), is a recently identified, rare genomic disorder. The patients are affected by infantile hypotonia, poor growth and developmental delay. Facial dysmorphism may not be obvious in some patients. PTLS is associated with microduplication at chromosome 17p11.2. In the current study, three Korean patients are reported with their clinical and genetic features.

Materials and Methods: The clinical findings of each patient were reviewed. Karyotyping and multiplex ligation-dependent probe amplification (MLPA) analyses were done for genetic diagnoses.

Results: All the patients did not have the characteristic dysmorphic features, such as broad forehead, triangular face, asymmetric smile and palpebral fissures. On the other hand, all three patients were affected by variable degree of developmental delay, poor oral intake, failure to thrive, and language development disorders. Chromosome 17p11.2 duplication was identified by conventional karyotyping analysis only in one patient, whereas the other confirmed by MLPA analyses.

Conclusion: Delayed development was mostly commonly observed in our patients without distinct dysmorphic facial features. In this respect, genomic screening in patients with developmental delay would identify more cases with PTLS to understand their long-term clinical courses with the development of adequate psychological and rehabilitation education program.

Key words: Potocki-Lupski syndrome, Chromosome duplication, Developmental disabilities.

\section{Introduction}

Potocki-Lupski syndrome (PTLS) (OMIM \#610883) is a genetic disorder caused by the genomic duplication at chromosome 17p11.2 [1]. The region includes retinoic acid-induced protein 1 (RA/1) gene. Although the role of this gene is elusive, it has been suggested as associated with the psychiatric disorders such as developmental delay, schizophrenia and autism [2].

PTLS patients show the characteristic dysmorphic features such as microcephaly, prominent forehead, ears and nose, and broad square-shaped face [3]. In addition, most patients have multi-systemic problems including hypotonia in infancy, cardiovascular disorder, hearing impairment and failure to thrive (FT) due to feeding problem $[3,4]$. Among these, the neurodevelopmental problems are most commonly observed, including developmental delay, intellectual disability, autism and behavioral problems such as hyperactivity [3,5].

Most PTLS patients have $3.7 \mathrm{Mb}$ duplication at 17p11.2, with

\footnotetext{
Received: 29 July 2019, Revised: 29 August 2019, Accepted: 11 September 2019, Published: 31 December 2019

*Corresponding author: Han-Wook Yoo, M.D., Ph.D. (iD https://orcid.org/0000-0001-8615-186X

Department of Pediatrics, Asan Medical Center Children's Hospital, University of Ulsan College of Medicine, 88 Olympic-ro 43-gil, Songpa-gu, Seoul 05505, Korea.

Tel: +82-2-3010-3374, Fax: +82-2-473-3725, E-mail: hwyoo@amc.seoul.kr

Conflict of interest: The authors declare that they do not have any conflicts of interest.

(c) This is an open-access article distributed under the terms of the Creative Commons Attribution Non-Commercial License (http://creativecommons.org/licenses/by-nc/4.0/) which permits unrestricted non-commercial use, distribution, and reproduction in any medium, provided the original work is properly cited.

(c) Copyright 2019 by the Korean Society of Medical Genetics and Genomics www.e-kjgm.org
} 
larger and smaller duplications reported [5]. The heterogeneity of clinical manifestations are associated with the size of the duplicated genome [6].

Here we describe the three Korean patients with PTLS due to a duplication of 17p11.2 The clinical features of our patient will help to understand this rare genetic condition.

\section{Materials and Methods}

\section{Patient}

From June 2014 to December 2018, three Korean patients were diagnosed with PTLS in Asan Medical Center Children's Hospital, Seoul, Korea. We reviewed the clinical and genetic features of each patient, including sex, age at diagnosis, congenital anomalies, growth and development. This study was approved by the Institutional Review Board at the Asan Medical Center (approval number: 2019-0831), and appropriate written informed consent was obtained from the parents of each patient.

\section{Genetic testing}

Karyotyping was performed using each patient's peripheral blood sample by Giemsa-banded techniques at the level of 550 bands in metaphase. In order to identify the multiple microdeletion of the patients, multiplex ligation-dependent probe amplification (MLPA) analysis were done using two types of SALSA Reference Kit, P064 and P245 (MRC Holland, Amsterdam, The
Netherlands) according to the manufacturer's instructions. This SALSA MLPA probemix P064 Microdeletion Syndromes-1B contains 52 MLPA probes with amplification products, respectively, corresponding to the RA/1 and TOM1L2 genes located the 17p11.2 regions. This SALSA MLPA probemix P245 Microdeletion Syndrome-1A contains 50 MPLA probes with amplification products, respectively, corresponding to the $R A / 1, D R C 3$ and LLGL1 genes located at the 17p11.2 regions. In order to confirm PTLS, the fluorescence in situ hybridization (FISH) was conducted as hybrid probe for RA/1 locus at 17p11.2 and PAFAH1B1 (LIS1) gene at 17p13.3 in more than 10 metaphase and 200 interphase peripheral leukocytes.

\section{Results}

The clinical and genetic findings of each patient are summarized in Table 1.

\section{Case 1}

The patient was the second baby of non-consanguineous Korean parents. His prenatal period was uneventful. He was born after 39 weeks of gestation with 2,930 $\mathrm{g}$ of birth weight (standard deviation [SD] score, -0.7). His height was $49 \mathrm{~cm}$ (SD score, -0.3) and head circumference was $31 \mathrm{~cm}$ (SD score, -2). At birth, he was hypotonic and tachypneic. Echocardiogram, abdominal and brain ultrasonography were normal. His face was not dysmor-

Table 1. Clinical and genetic findings of patients with Potocki-Lupski syndrome

\begin{tabular}{|c|c|c|c|}
\hline Variable & Case 1 & Case 2 & Case 3 \\
\hline Age (at diagnosis) & $6 \mathrm{mo}$ & 1 yr 2 mo & 4 yr 7 mo \\
\hline Sex & Male & Male & Male \\
\hline Gestational age & 39 weeks & Full term & 40 weeks \\
\hline Birth weight (g) & 2,930 & No information & 2,600 \\
\hline Dysmorphic features & $\begin{array}{l}\text { Not dysmorphic, } \\
\text { mild camptodactyly }\end{array}$ & $\begin{array}{l}\text { Posteriorly rotated, } \\
\text { large ears, micrognathia }\end{array}$ & Not dysmorphic \\
\hline Cardiac & No & No evaluation & No evaluation \\
\hline Gastrointestinal & No & No & No \\
\hline Seizure & No & No & No \\
\hline Developmental delay & Yes & Yes & Yes \\
\hline Endocrinologic & Hyperthyrotropinemia & Not conducted & Not conducted \\
\hline Hearing \& ophthalmologic & Strabismus & No & No \\
\hline Skeletal & No & No & No \\
\hline Genitourinary & No & No & No \\
\hline Results of MLPA analysis & $\begin{array}{l}\text { 17p11.2 } \\
\text { rsa17p11.2(P064)x3 }\end{array}$ & $\begin{array}{l}\text { 17p11.2 } \\
\text { rsa17p11.2(P064)x3 }\end{array}$ & $\begin{array}{l}\text { 17p11.2 } \\
\text { rsa17p11.2(P064)×3 }\end{array}$ \\
\hline Karyotype & $46, X Y$ & $46, X Y$ & 46, XY, dup(17)(p11.2p11.2) \\
\hline
\end{tabular}

MLPA, multiplex ligation-dependent probe amplification. 
phic except mild camptodactyly. When he visited our clinic at 6 months at age, his weight was $6.3 \mathrm{~kg}$ (SD score, -2.1), height was $67.3 \mathrm{~cm}$ (SD score, -0.2 ) and head circumference was 42 $\mathrm{cm}$ (SD score, -1.1). Brain magnetic resonance image (MRI) did not show any remarkable abnormality. The thyroid stimulation hormone was mildly elevated in his thyroid function test. At 6 months of age, he was able to control his head, but not able to roll over. His sucking power was weak. At 20 months of age, he was able to stand with support. He had a surgical correction for strabismus at age 2 when he started to walk. At the last visit, he was able to say only 2 words ("mama", "dada") but to understand receptive language at until 3.

Genetic testing was performed to identify the cause of delayed growth and development. His karyotype was 46, XY. 17p11.2 duplication was identified by MLPA analysis (Fig. 1).

\section{Case 2}

The patient was the first baby of non-consanguineous Korean parents. He was born at full term. Perinatal and postnatal periods were uneventful. At 11 months of age, he was not able to speak meaningful word. Brain MRI study did not demonstrate any remarkable abnormality. The thyroid function test was not performed in our clinic.

When he visited out clinic at 14 months of age, he weight was $8 \mathrm{~kg}$ (SD score, -1.9), his height was $76.9 \mathrm{~cm}$ (SD score, 0) and head circumference was $45 \mathrm{~cm}$ (SD score, -1.0$)$. He was able to crawl but could not walk. He was still unable to say any meaningful word. Mild dysmorphic facial features such as posteriorly rotated, large ear, micrognathia were noted.

His karyotype was 46, XY. MLPA analysis suggested the presence of 17p11.2 duplication, which was confirmed by FISH

\section{A-1}

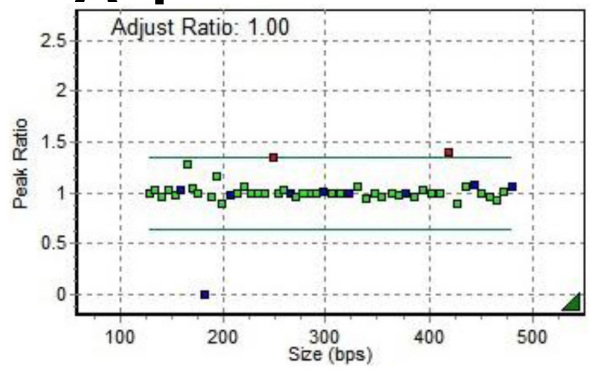

\section{B-1}
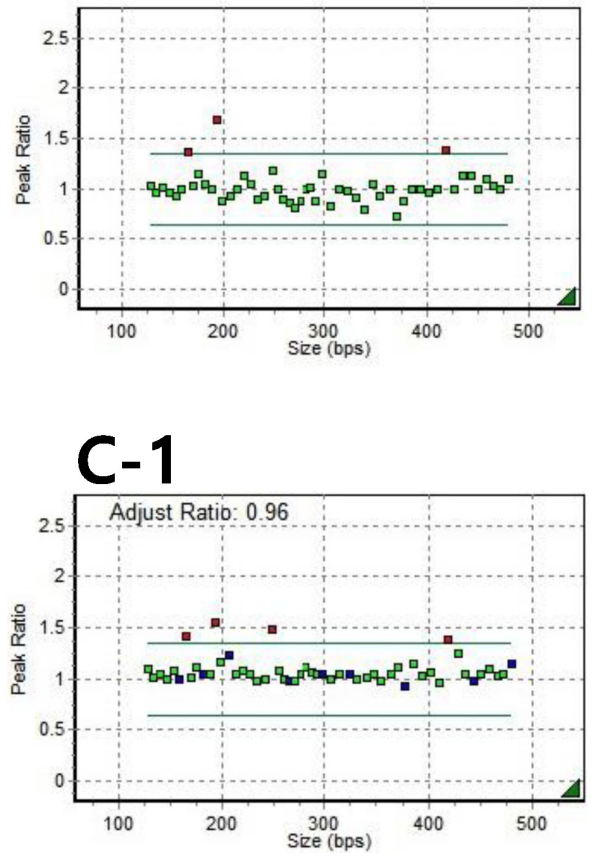

A-2

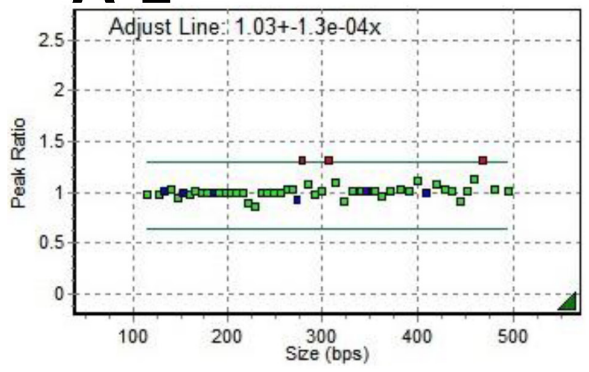

B-2

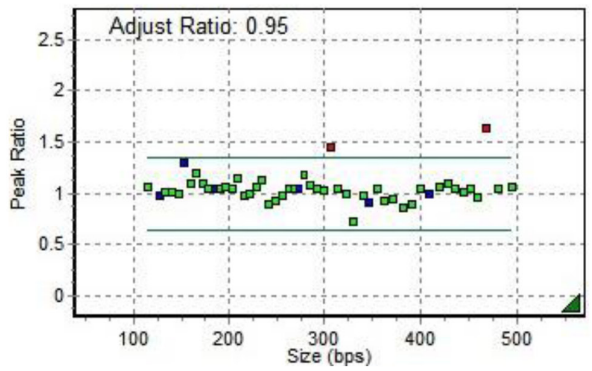

C-2

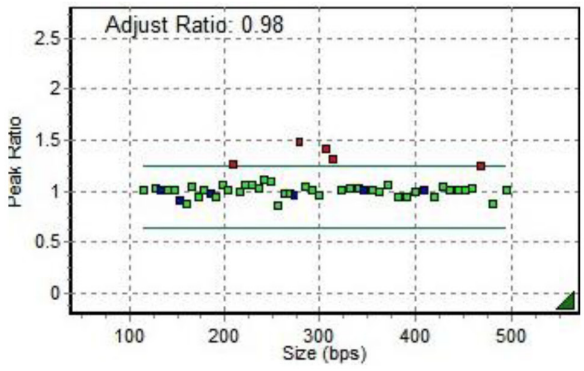

Fig. 1. The results of multiplex ligationdependent probe amplification (MLPA) analysis. MLPA analyses were done using two types of SALSA Reference Kit, P064 and P245 (MRC Holland) according to the manufacturer's instructions. P064 and 245 contains MLPA probes with amplification products, respectively, corresponding to RAl1, TOM1L2, DRC3 and LLGL1 genes located at the 17p11.2 regions. Green dots indicate normal allele, while red dots are abnormal that is duplicated at 17p11.2. (A) Case 1 patient, (B) case 2 patient, (C) case 3 patient. Each 1, 2: P064, P245 probemix. 

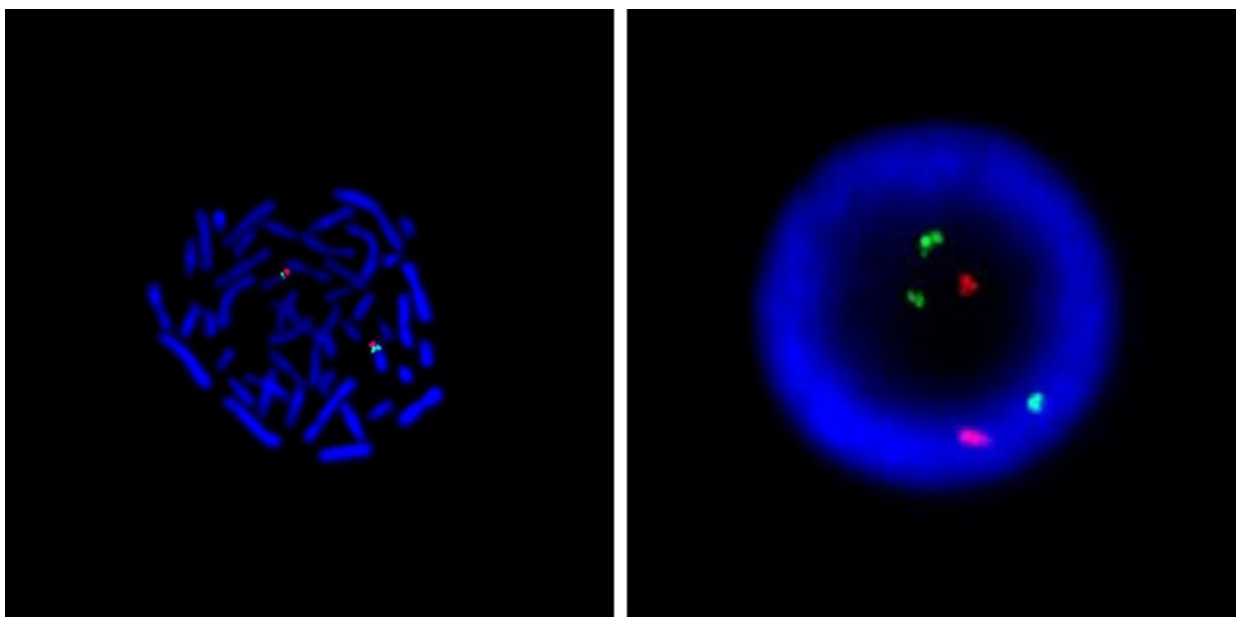

Fig. 2. The fluorescence in situ hybridization was performed with a hybrid probe for $R A / 1$ locus at 17p11.2 (green) and $P A$ FAH1B1 (LIS1) gene at 17p13.3 (red) (Case 2). Two duplication $R A / 1$ signals (green) were observed in a metaphase cell (left). In interphase cell, duplication $R A / 1$ signals (green) were more prominently visualized in chromatin before condensed (left). While there are 2 LIS signals as a normal control (red), there is one more $R A / 1$ signal (left).

analysis showing the duplicated RA/1 probe (Fig. 2).

\section{Case 3}

The patient was the first baby of non-consanguineous Korean parents. He was born after 40 gestational weeks via caesarean section with a birth weight of $2,600 \mathrm{~g}$ (SD score, -1.7). Facial dysmorphism was not noted nor was a remarkable congenital anomaly. Weaning of breast-milk feeding was delayed. At 18 months of age, he was able to walk alone. At 4 years of age, he is able to speak only a few meaningful words. At 4 year 7 months of age, he weight was $15 \mathrm{~kg}$ (SD score, -1.3) and height was $100.3 \mathrm{~cm}$ (SD score, -1.3). A Bayley developmental test at 52 months of age demonstrated that her cognitive development was at age 8 months, receptive communication at 6 months, expressive communication at 9 months, fine motor and gross motor development at 16 months and at 15 months, indicating profound global developmental delay. He presented a self-injurious habit of pulling his hair. At 4.8 years of age, he had severe intellectual disability and autism spectrum disorder. At 7 years of age, he can't speak more than 3 words phase. He has trouble interacting with other colleagues because of autism spectrum disorder. He has been taking psychiatric clinic treatment regularly.

His karyotype was 46, XY dup(17)(p11.2p11.2). 17p11.2 duplication was diagnosed by MLPA analysis at the same time.

\section{Discussion}

PTLS is a recently reported disorder, with the first genetically confirmed case reported by Brown et al. in 1996 [7]. The incidence of PTLS is thought to be at least 1 in 20,000 individuals. However, fewer than 50 cases have been described in the medi-
Table 2. Frequency of clinical features in three patients compared to those commonly reported in Potocki-Lupski syndrome

\begin{tabular}{lcc}
\hline \multicolumn{1}{c}{ Clinical features } & $\begin{array}{c}\text { Affected frequency } \\
\text { in the previous } \\
\text { reports }(n=58)\end{array}$ & $\begin{array}{c}\text { Number of } \\
\text { patients }(n=3)\end{array}$ \\
\hline Dysmorphic features & $25(43.1)$ & 1 \\
\hline Poor feeding in the neonatal period & $33(56.9)$ & 2 \\
Autism & $22(37.9)$ & 1 \\
\hline Failure to thrive & $20(34.5)$ & 3 \\
\hline Developmental delay & $52(89.7)$ & 3 \\
Cardiovascular involvement & $12(20.7)$ & 0 \\
\hline Seizure & $5(8.6)$ & 0 \\
\hline
\end{tabular}

Values are presented as number (\%) or number only.

cal literatures.

Most patients with PTLS have been described as showing global development delay [4]. All of our patients also had developmental problems including cognitive, motor and language delay. In addition, autism spectrum disorder has been observed in some patients with PTLS (37.9\%) [1,4], as in one of our patients (Table 2).

In recent reports, neuroimaging revealed mild brain abnormalities; mild attenuation of corpus callosum and delay in myelination and microcephaly $[4,8]$. In our patients, brain MRI at 0.5 to 1 year of age did not reveal any structural abnormalities (Table 2).

Seizure (8.6\%) has been rarely reported [4]. and it was not detected in our case, although longer-term observation is required (Table 2).

Facial dysmorphism such as broad forehead, palpebral fissures, bulbous nasal tip, posterior rotated ear and micrognathia have been reported (43.1\%), but our patients did not show easilynoticeable dysmorphism (Table 2). Only one patient had a mild facial anomaly including posteriorly rotated large ear, and mi- 
crognathia.

Feeding difficulty is another common manifestation in PTLS patients. This problem leads to FT and growth failure in 34\% to $71 \%$ of the patients $[1,9]$. Oropharyngeal incoordination may contribute to FIT in PTLS patients [9]. In our study, feeding problems were noted in 2 patients. Subsequently, most PTLS patients were consistently small in weight and height, indicating that the intrinsic genetic factors may underline these growth abnormality [9].

PTLS patients have a wide range of congenital multi-organ anomaly, such as cardiovascular anomaly (20.7\%), low thyroid stimulation hormone levels (30\%), ophthalmic, orthopedic, oropharyngeal, and renal anomalies $[4,8]$. None of our patients had a cardiac anomaly. One patient had strabismus and hearing loss was not reported in our study.

Duplicated region of chromosome 17p11.2 contains several genes including SMCR5, SREBF1, and TOM1L2. Among these, $R A / 1$ gene has been suggested as associated with the neurodevelopmental abnormality in PTLS patients. The RA/1 gene was first isolated in 1995 [10]. RA/1 is expressed in human hippocampus, cortex and cerebellum, and is suggested to be associated with cognitive and motor nervous function [2]. RAl1 is a transcription factor but its precise mode of action is unclear. On the other hand, when a subject has a heterozygous deletion at $17 p 11.2$ including the $R A / 1$ gene, facial dysmorphism, mental retardation and neurobehavioral disorders may develop, known as Smith-Magenis syndrome. Therefore, the $R A / 1$ gene may have a role in the development of human intellectual and behavioral skills by the gene-dosage effect $[2,11]$.

The common recurrent duplication of PTLS is heterozygous 3.7 Mb duplication at 17p11.2 (chr17:16,757,111-20,219,651 [hg 19]). The size of duplication region might be associated with the clinical severity of the PTLS patients [1].

For the identification of PTLS, karyotyping, FISH, MLPA analysis, and chromosome microarray (CMA) can be performed. In our study, FISH and MLPA performed to diagnosis this syndrome, but microarray is the most sensitive diagnostic tool that can reveal the duplication length $[4,12]$.

The limitation of this study is retrospective observation study on only a small number of patients. The duplication size was not determined since CMA analysis was not performed.

In conclusion, as PTLS is a rare genomic disorder associated with developmental delay but with mild dysmorphism, its clinical suspicion is not easy based on physical examination alone. Although most mutation occurs as a de novo microduplication at 17p11.2 and length of the duplication is $3.7 \mathrm{Mb}$ [4]. Inherited
PTLS is also rarely reported [12]. Universal screening with CMA for patients with delayed development will help to identify more cases, which will help to understand its long term prognosis and provide adequate psychological and rehabilitation education.

\section{Acknowledgements}

This research was supported in part by the Bio \& Medical Technology Development Program of the National Research Foundation (NRF) funded by the Korean government (NRF2018M3A9H1078335).

\section{References}

1. Potocki L, Bi W, Treadwell-Deering D, Carvalho CM, Eifert A, Friedman EM, et al. Characterization of Potocki-Lupski syndrome (dup(17)(p11.2p11.2)) and delineation of a dosage-sensitive critical interval that can convey an autism phenotype. Am J Hum Genet 2007;80:633-49.

2. Fragoso YD, Stoney PN, Shearer KD, Sementilli $A$, Nanescu SE, Sementilli $P$, et al. Expression in the human brain of retinoic acid induced 1 , a protein associated with neurobehavioural disorders. Brain Struct Funct 2015;220:1195-203.

3. Gulhan Ercan-Sencicek A, Davis Wright NR, Frost SJ, Fulbright RK, Felsenfeld $S_{1}$ Hart L, et al. Searching for Potocki-Lupski syndrome phenotype: a patient with language impairment and no autism. Brain Dev 2012;34:700-3.

4. Praticò $A D$, Falsaperla R, Rizzo R, Ruggieri M, Verrotti A, Pavone P. A new patient with Potocki-Lupski syndrome: a literature review. J Pediatr Genet 2018;7:29-34

5. Bissell S, Wilde L, Richards C, Moss J, Oliver C. The behavioural phenotype of Potocki-Lupski syndrome: a cross-syndrome comparison. J Neurodev Disord 2018;10:2.

6. Shuib S, Saaid NN, Zakaria Z, Ismail J, Abdul Latiff Z. Duplication 17p11.2 (Potocki-Lupski syndrome) in a child with developmental delay. Malays J Pathol 2017;39:77-81.

7. Brown A, Phelan MC, Patil S, Crawford E, Rogers RC, Schwartz C. Two patients with duplication of 17p11.2: the reciprocal of the SmithMagenis syndrome deletion? Am J Med Genet 1996;63:373-7.

8. Shchelochkov OA, Cheung SW, Lupski JR. Genomic and clinical characteristics of microduplications in chromosome 17. Am J Med Genet A 2010;152A:1101-10.

9. Soler-Alfonso C, Motil KJ, Turk CL, Robbins-Furman P, Friedman EM,

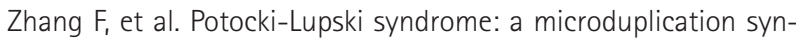
drome associated with oropharyngeal dysphagia and failure to thrive J Pediatr 2011;158:655-9.e2. 
10. Imai Y, Suzuki Y, Matsui T, Tohyama M, Wanaka A, Takagi T. Cloning of a retinoic acid-induced gene, GT1, in the embryonal carcinoma cell line P19: neuron-specific expression in the mouse brain. Brain Res Mol Brain Res 1995;31:1-9.

11. Carmona-Mora P, Walz K. Retinoic acid induced 1, RAl1: a dosage sensitive gene related to neurobehavioral alterations including autistic behavior. Curr Genomics 2010;11:607-17.

12. Magoulas PL, Liu P, Gelowani V, Soler-Alfonso C, Kivuva EC, Lupski JR, et al. Inherited dup(17)(p11.2p11.2): expanding the phenotype of the Potocki-Lupski syndrome. Am J Med Genet A 2014;164A:500-4. 